\title{
Tp-e interval and Tp-e/JT ratio before and after catheter ablation in patients with Wolff Parkinson White syndrome
}

\author{
(1)Ahmet Akdi' ${ }^{1}$, Özcan Özeke² \\ ${ }^{1}$ Ankara City Hospital, Department of Cardiology, Ankara, Turkey \\ ${ }^{2}$ University of Health Sciences, Ankara City Hospital, Department of Cardiology, Ankara, Turkey
}

Cite this article as: Akdi A, Özeke Ö. Tp-e interval and Tp-e/JT ratio before and after catheter ablation in patients with Wolff Parkinson White syndrome. J Health Sci Med 2022; 5(1): 291-296.

\begin{abstract}
Aim: Tp-e and Tp-e/QT are novel markers of ventricular repolarization. However, preexcitation in Wolff Parkinson White (WPW) syndrome may preclude accurate measurement of repolarization as assessed by the QT. We aimed to research the assessment of Tp-e and Tp-e/JT before and after ablation in WPW.

Material and Method: We enrolled 50 consecutive patients with symptomatic WPW syndrome who underwent catheter ablation of their accessory pathways for treatment. All of the patients were successfully ablated. ECGs were performed before the procedure and repeated after the ablation procedure.

Results: Tp-e ( $86.6 \pm 16.2$ vs $80.6 \pm 12.5 ; \mathrm{p}=0.021)$ and Tp-e(c) $(96.7 \pm 18.9$ vs $88.3 \pm 11.2 ; \mathrm{p}=0,005)$ showed a significant decrease after the procedure. Tp-e/JT $(0.307 \pm 0.053$ vs $0.279 \pm 0.040 ; \mathrm{p}=0.001)$ and Tp-e/JT(c) $(0.276 \pm 0.048$ vs $0.255 \pm 0.045 ; \mathrm{p}=0.009)$ showed a significant decrease after the procedure. There was a significant association between ERP and before procedure Tp-e (c) $(\mathrm{r}=-0.370, \mathrm{p}=0.008)$ and Tp-e/JT $(\mathrm{r}=-0.371, \mathrm{p}=0.008)$.

Conclusion: This study demonstrated that Tp-e and Tp-e/JT were significantly diminished in WPW after the ablation.

Keywords: Catheter ablation, JT interval, Tp-e interval, Tp-e/JT ratio, Wolff Parkinson White syndrome
\end{abstract}

\section{INTRODUCTION}

The Wolff-Parkinson-White (WPW) syndrome means to the presence of an overt accessory atrioventricular pathway, in combination with usually recurrent tachyarrhythmias, which may provoke ventricular tachyarrhythmias (VT) (1). In patients with WPW syndrome, the propagating impulse is conducted over the accessory pathway and causes ventricular preexcitation leading to typical alterations of ventricular depolarization and repolarization. Delayed ventricular repolarization has also been associated with VT (2).

Ventricular repolarization can be defined on ECG using QT interval, QT dispersion, and T-wave measurements. The QT interval represents both ventricular depolarization and repolarization, so it is dependent on the QRS duration. Several studies have shown that the Tp-e interval, the interval between the peak and the end of the $\mathrm{T}$ wave, is specified as an index of total dispersion of repolarization (3-5). Longer Tp-e interval may predict ventricular arrhythmias and mortality $(6,7)$. Therefore, the Tp-e/QT ratio was suggested to be a better marker of ventricular arrhythmias $(8,9)$.
Depolarization abnormalities in WPW patients may preclude accurate measurement of repolarization as assessed by the QT and corrected QT intervals. The JT and corrected JT (JT(c)) intervals particularly represent ventricular repolarization and have been shown to be independent of the ventricular depolarization and the QRS duration (10). Instead of the QT interval, the JT interval may be a more reliable measure of repolarization in WPW syndrome due to ventricular activation abnormalities. In this study, we aimed to research the assessment of Tp-e interval and Tp-e/JT ratio before and after catheter ablation in patients with WPW syndrome.

\section{MATERIAL AND METHOD}

The study was carried out with the permission of Ankara City Hospital, Clinical Researchs Ethics Committee (Date: 2021, Decision No: E1-12-2031). All procedures were carried out in accordance with the ethical rules and the principles of the Declaration of Helsinki. 


\section{Study Population}

In this study, we retrospectively enrolled 50 consecutive patients with symptomatic WPW syndrome who underwent catheter ablation of their accessory pathways for treatment. All of the patients were successfully ablated. Patients who have either an atrial fibrillation/ flutter, or permanent pacemaker therapy, or a complete/ incomplete bundle branch block were excluded.

\section{Catheter Ablation Procedure}

Prior to the procedure, all antiarrhythmic agents were withdrawn at least five half-lives. After vascular access was gained via femoral veins, two diagnostic catheters were placed in the right ventricle and coronary sinus for the electrophysiological study. Programmed atrial and ventricular stimulation was performed to induce atrioventricular reentrant tachycardia and to measure the effective refractory period (ERP) of the accessory pathway. In patients with left-sided pathways, a transseptal puncture was performed to access the left atrium. The ablation catheters were navigated under fluoroscopic and electroanatomic system guidance (Carto 3, Johnson and Johnson, USA). Programmed atrial and ventricular stimulation was performed to confirm the diagnosis of WPW, induce atrioventricular reentrant tachycardia, and prove the presence of an additional pathway, as well as to localize the exact location of the accessory pathways, as described in the literature (11). Directly before ablation, the bundle signal was marked using an ablation catheter on a three-dimensional (3D) map. Irrigated tip ablation was used while delivering radiofrequency energy. The catheter ablation was defined as a success when provided no signs of arrhythmia were documented within 15 minutes after the procedure.

\section{Electrocardiography}

The 12-leadECG was recorded at a paper speed of $50 \mathrm{~mm} / \mathrm{s}$ (Hewlett Packard, Page-writer, CA, USA) in the supine position. ECGs were performed before the procedure and repeated again after the ablation procedure. ECG measurements of JT and Tp-e intervals were performed by two cardiologists who were blinded to the patient data. An average value of three readings was calculated for each lead. All measurements were made manually from the same lead (Figure 1). The QT interval was measured from the beginning of the QRS complex to the end of the $\mathrm{T}$ wave and the JT interval was measured from the endpoint (J point) of the QRS complex to the end of the T wave. The Tp-e interval was defined as the interval from the peak of $\mathrm{T}$ wave to the end of $\mathrm{T}$ wave. The QT, the JT and the Tp-e intervals were corrected for the heart rate using the Bazett formula: $\mathrm{QT}(\mathrm{c})=\mathrm{QT} \sqrt{ }(\mathrm{R}-\mathrm{R}$ interval). The Tp-e/JT, the Tp-e/QT, the Tp-e/JT(c), the Tp-e/QT(c) ratios were calculated from these measurements.

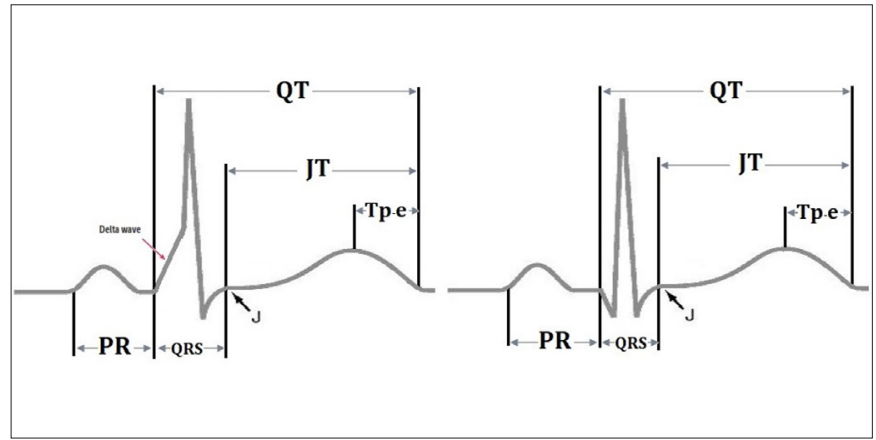

Figure 1. ECG measurements were made from the same lead as in the figure before and after catheter ablation.

\section{Statistical Analysis}

For statistical analysis, SPSS 22.0 Statistical Package Program for Windows (SPSS, Inc., IL, USA) was used. In order to test the normality of distribution Kolmogorov-Smirnov test was used. Quantitative variables with a normal distribution were specified as the mean \pm standard deviation and categorical variables were shown as number and percentage values. To determine the mean of the differences between two paired samples, Paired t-test, and Wilcoxon signedrank test was used. Categorical variables were compared with Mc Nemar test. The association between ERP and EKG parameters was evaluated by Pearson's correlation analysis. A p-value of $<0.05$ was accepted as statistically significant.

\section{RESULTS}

A total of 50 patients with symptomatic WPW syndrome who underwent catheter ablation were enrolled in our study. The clinical and baseline characteristics of the study population are shown in Table 1. The mean age of the study population was $35.7 \pm 15.1$ years, and $52.0 \%$ of patients were female, $16.0 \%$ of patients had hypertension, $4.0 \%$ of patients had diabetes mellitus and $4.0 \%$ of patients had coronary artery disease. Four of the patients were smoking and 9 of the patients were using an antiarrhythmic drug.

\begin{tabular}{|lc|}
\hline \multicolumn{2}{|l|}{ Table 1. Baseline characteristics of the study patients $(\mathrm{n}=50)$} \\
\hline Age, years & $35.7 \pm 15.1$ \\
Male, $\mathrm{n}(\%)$ & $24(48 \%)$ \\
Female, $\mathrm{n}(\%)$ & $26(52 \%)$ \\
Hypertension, $\mathrm{n}(\%)$ & $8(16 \%)$ \\
Diabetes mellitus, $\mathrm{n}(\%)$ & $2(4 \%)$ \\
Smoking, n (\%) & $4(8 \%)$ \\
Coronary artery disease, n (\%) & $4(8 \%)$ \\
Antiarrhythmic drug, $\mathrm{n}(\%)$ & $9(18 \%)$ \\
LVEF, \% & $59.2 \pm 6.3$ \\
ERP of AP, ms & $242.8 \pm 63.5$ \\
\hline $\begin{array}{l}\text { Data are given as mean } \pm \text { standard deviation, or n (\%). AP: accessory pathway; ERP: } \\
\text { effective refractory period; LVEF: Left ventricular ejection fraction }\end{array}$ \\
\hline
\end{tabular}


The electrocardiographic measurements of the study population before and after the procedure are shown in Table 2. Heart rate (75.4 \pm 11.2 vs $73.8 \pm 13.6$; $\mathrm{p}=0.382)$ was similar but PR (98.5 \pm 20.3 vs $153.1 \pm 27.4$; $\mathrm{p}<0.001)$, QRS (123.3 \pm 8.1 vs 94.3 \pm 10.9 ; p $<0.001)$, QT $(406.3 \pm 33.9$ vs $384.8 \pm 32.5 ; \mathrm{p}<0.001)$ and $\mathrm{QT}(\mathrm{c})(452.6 \pm 32.7$ vs $422.5 \pm 25.5 ; \mathrm{p}<0.001)$ are significantly different as expected before and after the procedure. Tp-e $(86,6 \pm 16,2$ vs $80.6 \pm 12.5 ; \mathrm{p}=0.021)$ and $\mathrm{Tp}-\mathrm{e}(\mathrm{c}) \quad(96.7 \pm 18.9$ vs $88.3 \pm 11.2 ; \mathrm{p}=0.005)$ intervals showed a significant decrease (Figure 2) while JT (283.0 \pm 33.1 vs 290.5 \pm 30.9 ; $\mathrm{p}=0.115)$ and $\mathrm{JT}(\mathrm{c})(314.9 \pm 31.2$ vs $318.6 \pm 23.9 ; \mathrm{p}=0.423)$ intervals were statistically similar after the procedure.

\begin{tabular}{|lccc|}
\hline $\begin{array}{l}\text { Table 2. Electrocardiographic interval measurements before and } \\
\text { after procedure }\end{array}$ & $\begin{array}{c}\text { Before } \\
\text { procedure }\end{array}$ & $\begin{array}{c}\text { After } \\
\text { procedure }\end{array}$ & $\begin{array}{c}\text { p } \\
\text { value }\end{array}$ \\
\hline Parameters & $75.440 \pm 11.202$ & $73.820 \pm 13.621$ & 0.382 \\
\hline Heart rate, bpm & $98.500 \pm 20.310$ & $153.100 \pm 27.459$ & $<0.001$ \\
PR interval, ms & $123.300 \pm 8.057$ & $94.300 \pm 10.974$ & $<0.001$ \\
QRS, ms & $406.300 \pm 33.924$ & $384.800 \pm 32.544$ & $<0.001$ \\
QT interval, ms & $283.000 \pm 33.120$ & $290.500 \pm 30.876$ & 0.115 \\
JT interval, ms & $86.600 \pm 16.208$ & $80.600 \pm 12.521$ & 0.021 \\
Tp-e interval, ms & $452.606 \pm 32.708$ & $422.533 \pm 25.522$ & $<0.001$ \\
$\begin{array}{l}\text { QT(c) interval, ms } \\
\text { JT(c) interval, ms }\end{array}$ & $314.947 \pm 31.185$ & $318.634 \pm 23.901$ & 0.423 \\
Tp-e(c) interval, ms & $96.660 \pm 18.900$ & $88.294 \pm 11.161$ & 0.005 \\
\hline $\begin{array}{l}\text { Data are given as mean } \pm \text { standard deviation, or } \mathrm{n}(\%) \text { Tp-e: T-peak to T-end interval, } \\
\text { terminal part of the QT interval, Tp-e(c): corrected T-peak to T-end interval; JT: J } \\
\text { point to T-end interval. }\end{array}$ & \multicolumn{4}{l}{} & \\
\hline
\end{tabular}

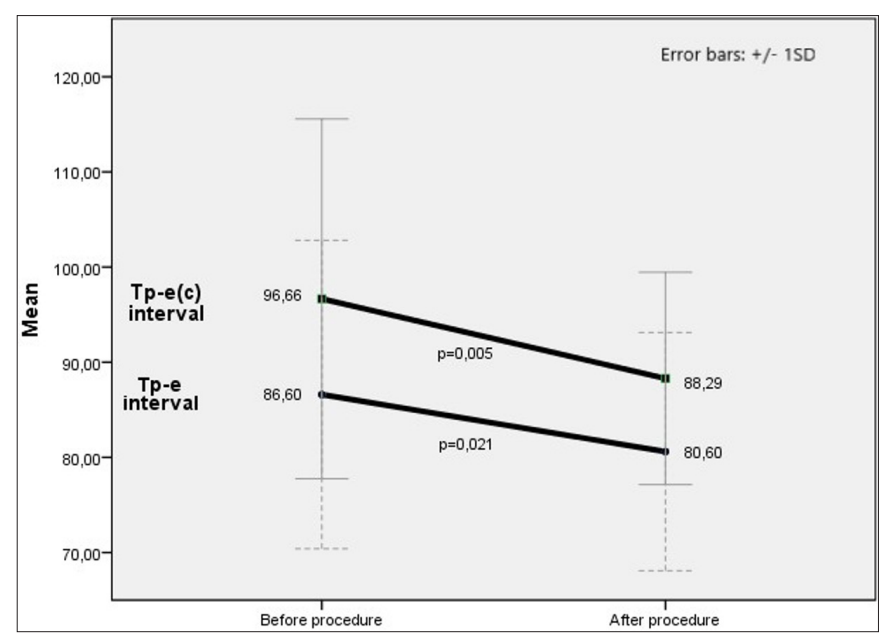

Figure 2. Tp-e and corrected Tp-e intervals showed a significant decrease after catheter ablation.

Calculated electrocardiographic interval ratios of study population before and after the procedure are shown in Table 3. While Tp-e/QT $(0.213 \pm 0.037$ vs $0.210 \pm 0.030$; $\mathrm{p}=0.549)$ and Tp-e/QT(c) (0.192 \pm 0.035 vs $0.192 \pm 0.035$; $\mathrm{p}=0.957)$ ratios did not show a significant change, Tp-e/ JT (0.307 \pm 0.053 vs $0.279 \pm 0.040 ; \mathrm{p}=0.001)$ and Tp-e/JT(c) $(0.276 \pm 0.048$ vs $0.255 \pm 0.045 ; \mathrm{p}=0.009)$ ratios showed a significant decrease (Figure 3 ). Tp-e/JT and Tp-e(c)/ JT(c) ratios were identical.
Table 3. Calculations of electrocardiographic interval ratio before and after procedure.

\begin{tabular}{|lccc|}
\hline Parameters & $\begin{array}{c}\text { Before } \\
\text { procedure }\end{array}$ & $\begin{array}{c}\text { After } \\
\text { procedure }\end{array}$ & p value \\
\hline Tp-e/QT ratio & $0.213 \pm 0.037$ & $0.210 \pm 0.030$ & 0.549 \\
Tp-e/QT(c) ratio & $0.192 \pm 0.035$ & $0.192 \pm 0.035$ & 0.957 \\
Tp-e/JT ratio & $0.307 \pm 0.053$ & $0.279 \pm 0.040$ & 0.001 \\
Tp-e/JT(c) ratio & $0.276 \pm 0.048$ & $0.255 \pm 0.045$ & 0.009 \\
Tp-e(c)/JT(c) ratio & $0.307 \pm 0.053$ & $0.279 \pm 0.040$ & 0.001 \\
\hline $\begin{array}{l}\text { Data are given as mean } \pm \text { standard deviation or n (\%). Tp-e: T-peak to T-end interval, } \\
\text { terminal part of the QT interval, Tp-e(c): corrected T-peak to T-end interval; JT: J } \\
\text { point to T-end interval }\end{array}$ & \multicolumn{4}{l}{} \\
\hline
\end{tabular}

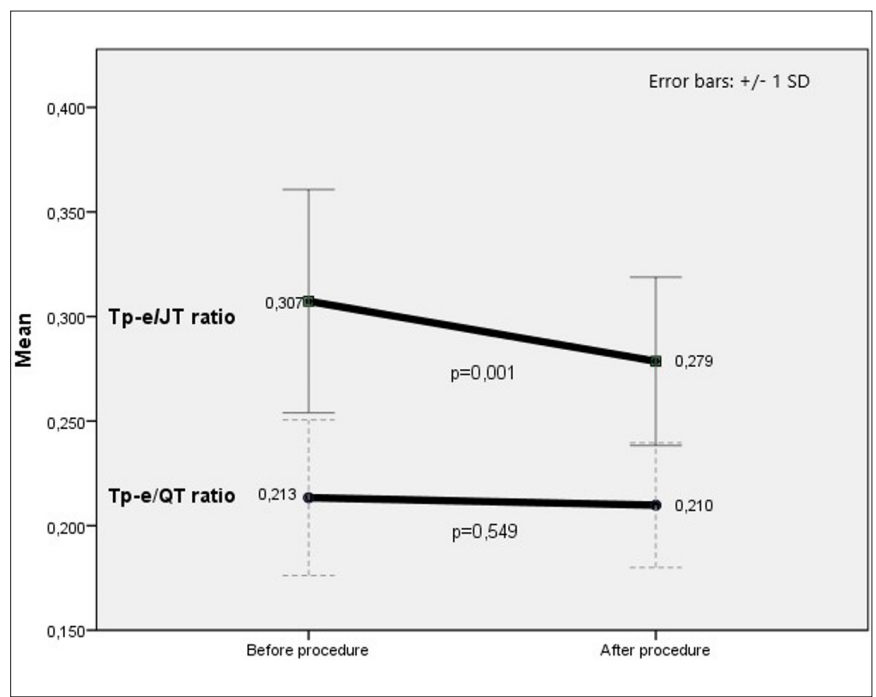

Figure 3. While Tp-e/QT ratio did not show a significant change, $\mathrm{Tp}-\mathrm{e} / \mathrm{JT}$ ratio showed a significant decrease.

Correlation analysis between ERP of the accessory pathway with ECG parameters are shown in Table 4. There was a significant association between ERP of the accessory pathway and before procedure Tp-e interval $(\mathrm{r}=-0.374, \mathrm{p}=0.007), \mathrm{Tp}-\mathrm{e} \quad(\mathrm{c})$ interval $(\mathrm{r}=-0.370$, $\mathrm{p}=0.008$, Figure 4a), Tp-e/JT $\operatorname{ratio}(r=-0.371, p=0.008$, Figure 4b).

Table 4. Correlation analysis between ERP of the AP and ECG parameters

\begin{tabular}{|lcc|}
\hline Parameters & $\begin{array}{c}\text { Correlation } \\
\text { coefficient (r) }\end{array}$ & p-value \\
\hline Tp-e interval before procedure & -0.374 & 0.007 \\
Tp-e interval after procedure & -0.214 & 0.135 \\
Tp-e (c) interval before procedure & $-0,370$ & 0.008 \\
Tp-e (c) interval after procedure & -0.105 & 0.467 \\
Tp-e/JT ratio before procedure & -0.371 & 0.008 \\
Tp-e/JT ratio after procedure & -0.143 & 0.320 \\
\hline $\begin{array}{l}\text { AP: accessory pathway; ERP: effective refractory period; Tp-e: T-peak to T-end } \\
\text { interval, terminal part of the QT interval, Tp-e(c): corrected T-peak to T-end interval; } \\
\text { JT: J point to T-end interval. }\end{array}$ \\
\hline
\end{tabular}



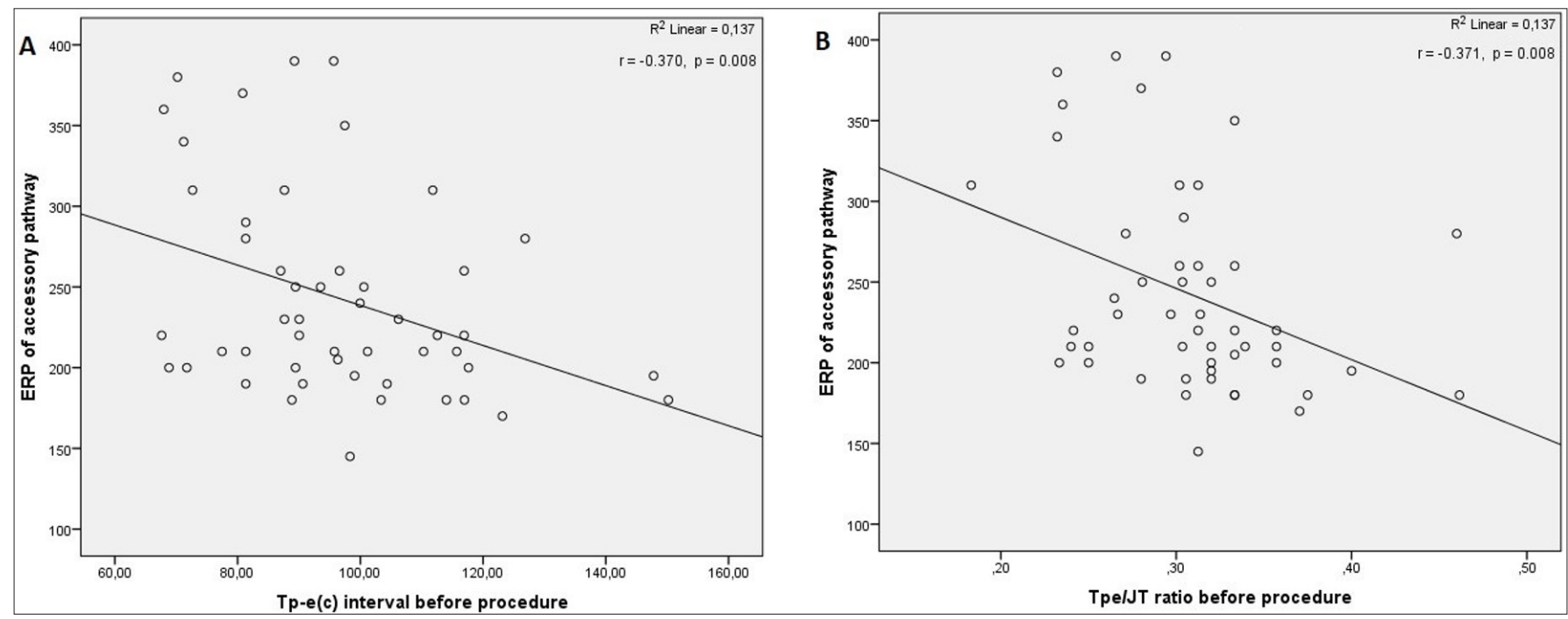

Figure 4. The correlation between effective refractory period (ERP) of the accessory pathway and (A) corrected Tp-e interval, (B) Tp-e/JT ratio before procedure was significant.

\section{DISCUSSION}

In the present study, we demonstrated that Tp-e interval and Tp-e/JT ratios were significantly diminished in patients with WPW syndrome after the catheter ablation procedure. This is the first study that demonstrated the relationship between Tp-e interval and Tp-e/JT ratio in patients with WPW syndrome.

In patients with WPW syndrome, it was previously shown that QT and QT(c) were significantly different when the post-ablation ECG was compared to the preablation one, whereas the JT and JT(c) values were unchanged (12). A previous study showed that eccentric ventricular depolarization due to preexcitation did not result in abnormal JT dispersion $(12,13)$. Our study has shown that eccentric ventricular depolarization due to preexcitation does not also result in abnormal JT interval, but it results in longer Tp-e interval which is specified as an index of total dispersion of repolarization $(4,14)$.

Many studies have shown longer Tp-e interval to be associated with higher risk. For example, prolonged Tp-e has been shown to be associated with increased mortality in patients with long QT syndrome (15), and similar findings have been reported in patients undergoing primary percutaneous coronary intervention for myocardial infarction (16). Prolonged Tp-e has been shown to predict VT, sudden cardiac death, or both in patients with systolic dysfunction and implantable cardioverter defibrillators implanted for primary prevention (17). The relationship of longer Tp-e interval with VT also has been described extensively in patients with Brugada syndrome (18). Recently a study showed that Tp-e interval, Tp-e/QT ratio were prolonged in COVID-19 patients with pneumonia (19).

Catheter ablation reduced the frequency of arrhythmic events in a prospective randomized clinical trial of patients with asymptomatic pre-excitation (20). Shortening of Tp-e interval after the ablation in patients with WPW syndrome may be one of the mechanisms explaining this reduction of arrhythmic events after ablation in asymptomatic patients because the longer of Tp-e interval indicates greater dispersion of ventricular repolarization and therefore increased susceptibility to electrical reentry that can cause ventricular tachyarrhythmia (21).

The assumed mechanism of sudden cardiac death and ventricular fibrillation (VF) is rapid stimulation of the ventricular myocardium due to atrial fibrillation rapidly conducted through the accessory pathway (22) however atrial fibrillation is not the only VF initiating arrhythmia as shown by the tachyarrhythmias documented prior to the occurrence of VF (23). We can suppose that preexcited atrial fibrillation with a fast ventricular response may cause VT due to prolongation of the Tp-e interval in WPW syndrome. The vulnerable period in the cardiac cycle, simultaneous with the downsloping portion of the $\mathrm{T}$ wave in the ECG, is a dangerous period for VT. So, a longer vulnerable period means a greater risk for arrhythmia. Similarly, short ERP is one of the risk factors in WPW. This study showed a significant negative relationship between Tp-e or Tp-e (c) and the ERP of the accessory pathway and supported this supposition.

In the early post-ablation period, the qualitative changes of the $\mathrm{T}$ wave morphology ( $\mathrm{T}$ wave memory) are observed. Although it is considered that $\mathrm{T}$ wave memory is associated with longer of repolarization in the early activated areas, repolarization changes at the late activated areas were ranging from shortening, minimal, or no change to significant action potential duration prolongation $(24,25)$. In addition, previous reports described these changes in the $\mathrm{T}$ wave vector without the changes in the repolarization duration $(26,27)$. Inconsistency in these findings is a subject of an ongoing 
discussion. This study showed that Tp-e and Tp-e(c) intervals showed a significant shortening while JT and JT(c) intervals were statistically similar in the early postablation period.

Tp-e/QT ratio has been proposed to be a marker of ventricular repolarization $(9,28)$ but, QRS wave abnormalities in WPW patients may preclude accurate measurement of repolarization as assessed by the QT intervals. Therefore, like this ratio, Tp-e/JT can be a marker of ventricular repolarization in WPW syndrome. It is shown that the JT interval such as the QT interval is heart rate (HR) dependent (29). Also, correcting the Tp-e interval using Bazett's formula ( Tp-e $(c)=T p-e$ interval $/ \sqrt{ }$ (RR interval) has improved the predictive value of this marker for SCA risk (30). Mathematically, if 'corrected Tp-e/corrected JT' ratio is simplified by the common factor ( $\sqrt{ } \mathrm{RR}$ interval) of the numerator and denominator, the Tp-e/JT ratio is obtained. Thus, the Tp-e/JT ratio remains constant despite dynamic physiological changes in $H R$, and it may be a ventricular repolarization index that is independent of HR. Once the use of Tp-e and Tp-e/ JT is more investigated, it may become valuable as a part of future multi-component risk prediction algorithms in WPW.

There are some limitations to this study. First, the study has a relatively small sample size. A larger sample size would have strengthened our findings. Then, the relationship between ventricular arrhythmias and both Tp-e interval and Tp-e/JT ratio was not assessed in patients with WPW syndrome. Therefore, long-term follow-up and large-scale prospective studies are needed to investigate the predictive value of the Tp-e interval and Tp-e/JT ratio in patients with WPW syndrome.

\section{CONCLUSION}

We demonstrated that the pre-ablation Tp-e interval and Tp-e/JT ratios were significantly diminished in patients with WPW syndrome after the catheter ablation. Longterm follow-up and large-scale prospective studies are needed to investigate the predictive value of the Tp-e interval and Tp-e/JT ratio about mortality and malignant arrhythmias in patients with WPW syndrome.

\section{ETHICAL DECLARATIONS}

Ethics Committee Approval: The study was carried out with the permission of Ankara City Hospital, Clinical Researchs Ethics Committee (Date: 2021, Decision No: E1-12-2031).

Informed Consent: Because the study was designed retrospectively, no written informed consent form was obtained from patients

Referee Evaluation Process: Externally peer-reviewed.
Conflict of Interest Statement: The authors have no conflicts of interest to declare.

Financial Disclosure: The authors declared that this study has received no financial support.

Author Contributions: All of the authors declare that they have all participated in the design, execution, and analysis of the paper and that they have approved the final version.

\section{REFERENCES}

1. Bhatia A, Sra J, Akhtar M. Preexcitation syndromes. Curr Probl Cardiol 2016; 41: 99-137.

2. Cho JH, Leong $\mathrm{D}$, Cuk $\mathrm{N}$, et al. Delayed repolarization and ventricular tachycardia in patients with heart failure and preserved ejection fraction. PloS One 2021; 16: e0254641.

3. Kors JA, van Eck HJR, van Herpen G. The meaning of the Tp-Te interval and its diagnostic value. J Electrocardiol 2008; 41: 575-80.

4. Antzelevitch C, Sicouri S, Di Diego JM, et al. Does Tpeak-Tend provide an index of transmural dispersion of repolarization? Heart Rhythm 2007; 4: 1114-6.

5. Karaman K, Altunkaş F, Çetin M, et al. New markers for ventricular repolarization in coronary slow flow: Tp-e interval, Tp-e/QT ratio, and Tp-e/QTc ratio. Ann Noninvasive Electrocardiol 2015; 20: 338-44.

6. Castro Hevia J, Antzelevitch C, Tornés Bárzaga F, et al. TpeakTend and Tpeak-Tend dispersion as risk factors for ventricular tachycardia/ventricular fibrillation in patients with the Brugada syndrome. J Am Coll Cardiol 2006; 47: 1828-34.

7. Smetana P, Schmidt A, Zabel M, et al. Assessment of repolarization heterogeneity for prediction of mortality in cardiovascular disease: peak to the end of the $\mathrm{T}$ wave interval and nondipolar repolarization components. J Electrocardiol 2011; 44: 301-8.

8. Zhao X, Xie Z, Chu Y, et al. Association between Tp-e/QT ratio and prognosis in patients undergoing primary percutaneous coronary intervention for ST-segment elevation myocardial infarction. Clin Cardiol 2012; 35: 559-64.

9. Gupta P, Patel C, Patel H, et al. Tp-e/QT ratio as an index of arrhythmogenesis. J Electrocardiol 2008; 41: 567-74.

10.Zhou SH, Wong S, Rautaharju PM, Karnik N, Calhoun HP. Should the JT rather than the QT interval be used to detect prolongation of ventricular repolarization? J Electrocardiol 1992; 25: 131-6.

11. Ceresnak SR, Dubin AM, Kim JJ, et al. Success rates in pediatric WPW ablation are improved with 3-dimensional mapping systems compared with fluoroscopy alone: A multicenter study. J Cardiovasc Electrophysiol 2015; 26: 412-6.

12. Salim MA, Case CL, Gillette PC. The JT interval as a depolarization independent measurement of repolarization: lessons from catheter ablation of the Wolff-Parkinson-White syndrome. Pacing Clin Electrophysiol 1995; 18: 2158-62.

13. Tabatabaei P, Keikhavani A, Haghjoo M, et al. Assessment of QT and JT Intervals in Patients With Left Bundle Branch Block. Res Cardiovasc Med 2016; 5: e31528.

14. Kors JA, van Eck HJR, van Herpen G. The meaning of the Tp-Te interval and its diagnostic value. J Electrocardiol 2008; 41: 575-80.

15. Topilski I, Rogowski O, Rosso R, et al. The morphology of the QT interval predicts torsade de pointes during acquired bradyarrhythmias. J Am Coll Cardiol 2007;49: 320-8.

16. Haarmark C, Hansen PR, Vedel-Larsen E, et al. The prognostic value of the Tpeak-Tend interval in patients undergoing primary percutaneous coronary intervention for ST-segment elevation myocardial infarction. J Electrocardiol 2009; 42: 555-60. 
17. Rosenthal TM, Stahls PF, Abi Samra FM, et al. T-peak to T-end interval for prediction of ventricular tachyarrhythmia and mortality in a primary prevention population with systolic cardiomyopathy. Heart Rhythm 2015; 12: 1789-97.

18.Letsas KP, Weber R, Astheimer K, Kalusche D, Arentz T. TpeakTend interval and Tpeak-Tend/QT ratio as markers of ventricular tachycardia inducibility in subjects with Brugada ECG phenotype. Europace 2010; 12: 271-4.

19. Keleşoğlu Ş, Yllmaz Y. Assessment of Tp-e interval, Tp-e/QT, and Tp-e/QTc ratios, in patients with COVID-19 infected with or without pneumonia. J Health Sci Med 2021; 4: 615-21.

20. Pappone C, Santinelli V, Manguso F, et al. A randomized study of prophylactic catheter ablation in asymptomatic patients with the Wolff-Parkinson-White syndrome. New Engl J Med 2003; 349: 1803-11.

21. Antzelevitch C. Cellular basis and mechanism underlying normal and abnormal myocardial repolarization and arrhythmogenesis. Ann Med 2004; 36: 5-14.

22. Delise P, Sciarra L. Sudden Cardiac Death in Patients with Ventricular Preexcitation. Card Electrophysiol Clin. 2020; 12 519-25.

23. Aranyo J, Bazan V, Rueda F, Sarrias A, Bisbal F, Villuendas R. Ventricular fibrillation in a patient with Wolff-ParkinsonWhite syndrome unrelated to pre-excited atrial fibrillation. Ann Noninvasive Electrocardiol 2019; 24: e12662.

24.Spragg DD, Akar FG, Helm RH, Tunin RS, Tomaselli GF, Kass DA. Abnormal conduction and repolarization in late-activated myocardium of dyssynchronously contracting hearts. Cardiovasc Res 2005; 67: 77-86.

25. Jeyaraj D, Wilson LD, Zhong J, et al. Mechanoelectrical feedback as novel mechanism of cardiac electrical remodeling. Circulation 2007; 115: 3145-55.

26. Gunaseelan R, Sasikumar M, Aswin K, Nithya B, Balamurugan N, Vivekanandan M. Memory T-Waves, a Rare Cause of T-Wave Inversion in the Emergency Department. J Emerg Trauma Shock 2020; 13: 312-6.

27. Poole JE, Bardy GH. Further evidence supporting the concept of T-wave memory: observation in patients having undergone highenergy direct current catheter ablation of the Wolff-ParkinsonWhite syndrome. Eur Heart J 1992; 13: 801-7.

28.Zhao X, Xie Z, Chu Y, et al. Association between Tp-e/QT ratio and prognosis in patients undergoing primary percutaneous coronary intervention for ST-segment elevation myocardial infarction. Clin Cardiol 2012; 35: 559-64.

29. Hnatkova K, Johannesen L, Vicente J, Malik M. Heart rate dependency of JT interval sections. J Electrocardiol 2017; 50: 81424.

30. Chua KCM, Rusinaru C, Reinier K, et al. Tpeak-to-Tend interval corrected for heart rate: A more precise measure of increased sudden death risk? Heart Rhythm 2016; 13: 2181-5. 